Cite this: Phys. Chem. Chem. Phys., 2014, 16, 4639

Received 5th November 2013, Accepted 10th January 2014

DOI: $10.1039 / c 3 c p 54676 d$

\section{Fatty acid droplet self-division driven by a chemical reaction $\dagger$}

\author{
I. Derényi ${ }^{a}$ and I. Lagzi ${ }^{\star b}$
}

Division of a millimeter-sized and compartmentalized fatty acid droplet is triggered by a chemical pH clock reaction operating on the ten-second time scale. The autonomous $\mathrm{pH}$ change inside the compartment translates into the deprotonation of the fatty acid molecules, leading to a negative interfacial tension at the water-oil interface inside the droplet. This phenomenon induces the expansion of the droplet, which is followed by its division into daughter droplets governed by the Plateau-Rayleigh instability.

www.rsc.org/pccp

\section{Introduction}

Self-division of microscopic or macroscopic compartments (reverse micelles, vesicles or macroemulsions) has gained much interest in the past decades because of the importance of creating and engineering a minimal cell that can be considered a necessary link in the transition of inanimate matter to life. ${ }^{1-7}$ In these pioneering studies the self-division is governed by the instability of membranes containing surface active (e.g., fatty acid) molecules produced by chemical reactions occurring at the inner interface (e.g., basic hydrolysis of fatty acid anhydrides). These artificial compartments can serve as microsized reactors that support the replication of nucleic acids, ${ }^{6}$ and it has been shown that this process can be successfully coupled to self-reproduction of giant vesicles. ${ }^{7}$ DNA replication within giant vesicles and vesicle growth and division occur in two consecutive and distinct steps. ${ }^{7}$ After DNA replication the vesicle growth and division can be externally initiated by the addition of a membrane precursor to DNA-amplified giant vesicles. Despite these fascinating results, self-division of small artificial compartments (droplets, vesicles) by autonomous internal stimuli remains challenging. Here we show that the self-division of a compartmentalized fatty acid droplet at the air-water interface can be successfully controlled in an autonomous way using a chemical reaction ( $\mathrm{pH}$ clock reaction) operating on a ten-second time scale inside the compartment (Fig. 1). ${ }^{8-10}$ Experimental results are explained by a theoretical interpretation based on the Plateau-Rayleigh instability, and our statistical analysis

\footnotetext{
a ELTE-HAS "Lendulet" Biophysics Research Group, Department of Biological Physics, Eötvös Loránd University, Budapest H-1111, Hungary

${ }^{b}$ Department of Physics, Budapest University of Technology and Economics, Budapest H-1521, Hungary. E-mail: lagzi@vuk.chem.elte.hu

$\dagger$ Electronic supplementary information (ESI) available: Movie showing the selfdivision of a fatty acid droplet. See DOI: $10.1039 / \mathrm{c} 3 \mathrm{cp} 54676 \mathrm{~d}$
}

reveals that the most probable scenario for division is into two and equally sized droplets.

\section{Results and discussion}

The process of droplet self-division is illustrated in Fig. 1 . We placed a small drop $(\sim 80 \mu \mathrm{L})$ of the $1: 2(\mathrm{v} / \mathrm{v})$ mixture of 2-hexyldecanoic acid (HDA) (Sigma-Aldrich) and paraffin oil ${ }^{11}$ (Sigma-Aldrich) containing a small amount (ca. $5 \mathrm{mg} \mathrm{mL}{ }^{-1}$ ) of Oil Red (Sigma-Aldrich) dye for visualization in $\sim 4-5 \mathrm{~mm}$ deep DI water in a Petri dish. Then we carefully injected a smaller $(\sim 8 \mu \mathrm{L})$ drop from the premixed $\mathrm{pH}$ clock reaction solution

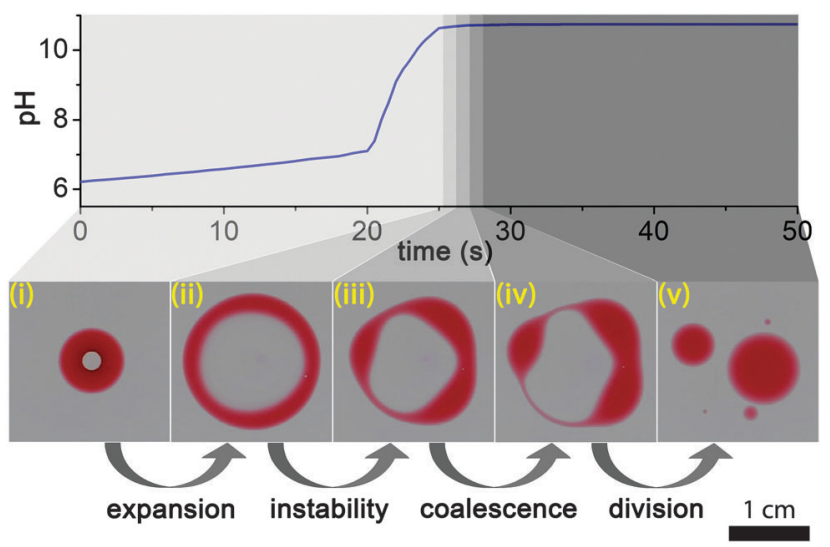

Fig. 1 Cycle of the self-division. A fatty acid droplet containing a water phase inside with a $\mathrm{pH}$ (sulfite-formaldehyde) clock reaction that lasts for several tens of seconds (i). When the $\mathrm{pH}$ inside the droplet changes and reaches basic $\mathrm{pH}$, the 2-hexyldecanoic acid (HDA) molecules become deprotonated, changing the surface tension inside the compartment and leading to the destabilization and expansion of the droplet (ii). The resulting toroidal (ring-like) structure becomes unstable and undergoes the PlateauRayleigh instability (iii) followed by the coalescence of some connected blobs (iv) and the division of the perturbed droplet (v). 
into the center of the floating organic droplet with a micropipette, thus creating an inner water phase compartment. We prepared fresh stock solutions of reagents for a clock reaction prior to the experiments: (i) sodium sulfite (Sigma-Aldrich)/ sodium bisulfite (Sigma-Aldrich) buffer solution $\left(\left[\mathrm{SO}_{3}{ }^{2-}\right]=\right.$ $0.010 \mathrm{M},\left[\mathrm{HSO}_{3}{ }^{-}\right]=0.10 \mathrm{M}$ ) and (ii) $0.2 \mathrm{M}$ formaldehyde (Sigma-Aldrich). ${ }^{12,13}$ Before the experiments, we mixed equal volumes of these stock solutions and immediately injected it into the center of the organic drop suspended in the air-water interface. This sulfite-formaldehyde clock reaction operates between neutral (slightly acidic) $\mathrm{pH}(\sim 6)$ and basic $\mathrm{pH}(\sim 10)$, and it can dramatically change the protonation rate of fatty acid molecules in this range, because the $\mathrm{p} K_{\mathrm{a}}$ of HDA is $\sim 8.3^{4}$ The process has two phases, the first, "low" $\mathrm{pH}$, phase is governed by the sulfite/bisulfite buffer which compensates any changes in $\mathrm{pH}$ up to $\mathrm{pH} \sim 7.5$ in the methylene glycol-sulfite autocatalytic reaction (Fig. 1). The second phase starts when all sulfite has been consumed, which is followed by an autocatalytic evolution of $\mathrm{OH}^{-}$ producing a fast $\mathrm{pH}$ change up to $\mathrm{pH} \sim 10$ ("high" $\mathrm{pH}$ state)..$^{8-10}$

After the aqueous solution containing the $\mathrm{pH}$ clock reaction had been injected into the organic drop, it formed a stable compartment, lasting for about $20 \mathrm{~s}$ (induction period). This long stability indicates that the compartment was fully engulfed by the droplet, covered with a thin organic layer from below and above (Fig. 1).

As Patashinski et al. demonstrated, ${ }^{14}$ a chemical reaction between two non-miscible liquids can rapidly create surfactants directly at the interface, and this reaction-driven overpopulation can make the effective interfacial surface tension negative. This phenomenon was exemplified by the deprotonation of HDA molecules at the interface between a non-polar phase and the surrounding aqueous medium containing a strong base. Similarly, when the $\mathrm{pH}$ inside our compartment changed from neutral $(\mathrm{pH} \sim 6.0-8.0)$ to basic range $(\mathrm{pH} \sim 10.0)$ more and more HDA molecules migrated to the inner interface and became deprotonated, resulting in a negative effective surface tension, thus destabilizing and expanding the interface. This then led to the disruption of the thin organic layers below and above the compartment, transforming the spherical topology of the original droplet to a ring-like (toroidal) structure (Fig. 1 and Movie S1, ESI $\dagger$ ). As the aqueous space in the hole of the ring remained basic, containing dissolved deprotonated HDA surfactant molecules, the air-water interface in the hole must have retained a smaller surface tension than the outside, leading to a fast expansion of the ring. Note that there was a lag of about a second between the times when the $\mathrm{pH}$ clock reaction reached the highest value and when the expansion started, probably due to the deprotonation dynamics and the reorganization of the HDA molecules at the water-oil interface inside the droplet. We carried out control experiments injecting a drop of basic solution $(\mathrm{pH} \sim 10)$ into the organic droplet. The division started about a second later, showing that this phenomenon is indeed driven by a $\mathrm{pH}$ induced surface tension change.

The initial growth speed $(v)$ of the radius $(R)$ of the ring was of the order of $0.1 \mathrm{~ms}^{-1}$ which is consistent with a surface tension driven process. This can be verified by the following argument. The outward force acting on a small ( $\Delta l$ long), approximately cylindrical segment of the ring due to the surface tension difference $(\Delta \sigma)$ between the outside and the inside of the ring is $\Delta \sigma \Delta l$. This is balanced by the viscous drag force, which, at the high Reynolds number that characterizes the expansion $\left(\operatorname{Re}=v R / \eta \sim 10^{3}\right.$, where $\eta \sim 10^{-6} \mathrm{~m}^{2} \mathrm{~s}^{-1}$ is the kinematic viscosity of water), is $1 / 2 \rho v^{2} C h \Delta l$, where $\rho \sim 10^{3} \mathrm{~kg} \mathrm{~m}^{-3}$ is the density of water, $h$ is the height of the ring, and $C$ is the drag coefficient, which is of the order of unity $(C \sim 1.2$ for a long cylinder fully immersed in water). The height of the ring can be estimated from the volume $(V \sim 80 \mu \mathrm{L})$ of the droplet and the observed width $(w)$ of the ring as $h \sim V /(2 \pi R w)$. From the force balance one then gets the speed of the expanding ring as

$$
v \sim \sqrt{\frac{2 \Delta \sigma 2 \pi R w}{\rho C V}}
$$

which, for $R \sim 1 \mathrm{~cm}, w \sim 0.1 \mathrm{~cm}$, and $\Delta \sigma$ of the order of magnitude of $10 \mathrm{mN} \mathrm{m}^{-1}$ (confirmed by control experiments), is indeed about $0.1 \mathrm{~ms}^{-1}$.

The expansion of the ring must have terminated when the solution in the hole became diluted and its surface tension returned to that of pure water. From this moment on the shape of the ring-like organic drop became driven solely by the surface tension of the organic phase in pure water (below) and air (above). As a narrow ring can be considered as a slightly bent cylindrical object, it is expected to undergo the so-called Plateau-Rayleigh instability (best known from the breaking up of a stream of fluid into droplets), ${ }^{15,16}$ resulting in the self-division of the ring into

$$
N_{\text {init }} \sim 0.7 \frac{2 \pi R}{\pi w}=1.4 \frac{R}{w}
$$

small separated droplets. ${ }^{17}$ Although the number of initially appearing blobs on the ring in the experiments was consistent with this value (Fig. 2), several neighboring blobs remained connected to each other and then coalesced; thus the final number $(N)$ of the daughter droplets became somewhat smaller (Fig. 2).

We also investigated the efficiency of the $\mathrm{pH}$ clock reaction driven autonomous self-division, because not all $\mathrm{pH}$ cycles could trigger the self-division of the original (mother) droplet into two smaller parts. Fig. 3a shows the probability distribution of the number of daughter droplets formed after the selfdivision. Typically, the mother droplet is split into two daughter droplets with a high probability $(P=50 \%)$, which is the consequence of our protocol that leads to a torus with a geometric ratio $R / w$ mostly around 4 (see Fig. 2 ). The probability to produce more daughter droplets decreases and division into 6 or more droplets has not been observed in our 120 experiments. Interestingly, $10 \%$ of the experiments remained unsuccessful in the sense that the clock reaction could not induce division into at least two parts. Additionally, we examined the size ratio of the daughter droplets when exactly two of them were formed (Fig. 3b). We found that the most probable scenario is that the reaction driven self-division produces two similarly sized droplets, which is not surprising as both the Plateau-Rayleigh instability 


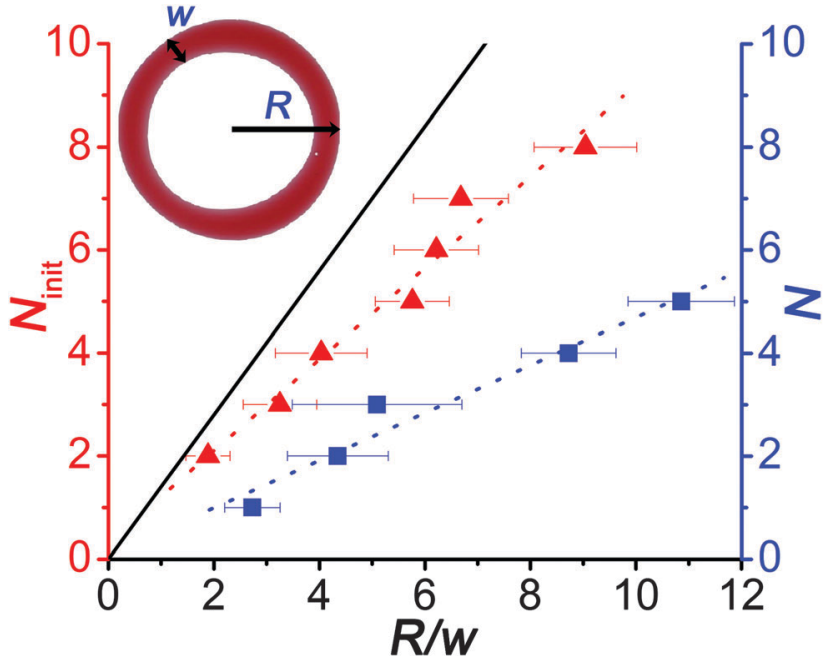

Fig. 2 Dependence of the number of initially appearing blobs/nodes $\left(N_{\text {init }}\right)$ due to the Plateau-Rayleigh instability and the number of the daughter droplets $(N)$ on the ratio of the radius $(R)$ and the width of the ring $(w)$ when the ring (toroid) has the highest spreading area. Solid line corresponds to the theoretical prediction given by eqn (1).
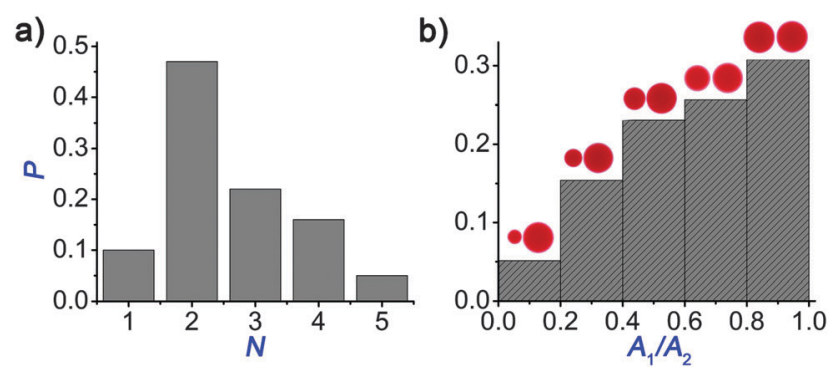

Fig. 3 Probability distribution of the number of the daughter droplets (a). The most probable scenario is the division into two droplets. Probability distribution of the size ratio of the droplets when two droplets are formed (b). The most probable event is the formation of the two and equally sized droplets.

produces uniform blobs and their subsequent coalescence occurs uniformly.

\section{Conclusions}

One of the key aspects of living cells is that they can self-divide in an autonomous way. We showed that such self-division can exist in an inanimate system. We constructed a simple millimeter-sized chemical object that can self-divide via a combination of nonlinear chemical dynamics and fatty acid chemistry. In our case this process is controlled by a chemical $\mathrm{pH}$ clock reaction, where $\mathrm{pH}$ change is translated into the change of the protonation of fatty acid head groups, inducing a surface tension driven self-division through the Plateau-Rayleigh instability. Obviously, this selfdivision does not repeat itself, as it would require both the growth of the daughter droplets and the reinitiation of the chemical reaction. Nevertheless, our experiments demonstrate that autonomous self-division of small droplets suspended in water can be achieved by a simple chemical reaction.
Division of a fatty acid droplet by a $\mathrm{pH}$ clock reaction is reminiscent of the division of a living cell, where the process is based on and driven by a biochemical nonlinear reaction network. It should be noted that the division of living cells is much more complex and has been shaped by millions of years of evolution. Yet, our observation can be considered as a step towards bridging the gap between the inanimate and animate world.

\section{Acknowledgements}

This research was supported by the Hungarian Research Foundation (OTKA K77908, K104666, K101436 and NK100296) and by the European Union and the State of Hungary, co-financed by the European Social Fund in the framework of TÁMOP 4.2.4. A/1-11-12012-0001 'National Excellence Program'.

\section{Notes and references}

1 J. W. Szostak, D. P. Bartel and P. L. Luisi, Nature, 2001, 409, 387-390.

2 M. M. Hanczyc, S. M. Fujikawa and J. W. Szostak, Science, 2003, 302, 618-622.

3 H. Takahashi, Y. Kageyama, K. Kurihara, K. Takakura, S. Murata and T. Sugawara, Chem. Commun., 2010, 46, 8791-8793.

4 K. P. Browne, D. A. Walker, K. J. M Bishop and B. A. Grzybowski, Angew. Chem., Int. Ed., 2010, 49, 6756-6759.

5 F. Caschera, S. Rasmussen and M. M. Hanczyc, ChemPlusChem, 2013, 78, 52-54.

6 S. Koga, D. S. Williams, A. W. Perriman and S. Mann, Nat. Chem., 2011, 3, 720-724.

7 K. Kurihara, M. Tamura, K. Shohda, T. Toyota, K. Suzuki and T. Sugawara, Nat. Chem., 2011, 3, 775-781.

8 R. McIlwaine, K. Kovács, S. K. Scott and A. F. Taylor, Chem. Phys. Lett., 2006, 417, 39-42.

9 K. Kovács, R. E. McIlwaine, S. K. Scott and A. F. Taylor, J. Phys. Chem. A, 2007, 111, 549-551.

10 K. Kovács, R. E. McIlwaine, S. K. Scott and A. F. Taylor, Phys. Chem. Chem. Phys., 2007, 9, 3711-3716.

11 Paraffin oil can be replaced by a natural vegetable oil (e.g., commercial sunflower oil - Venus ${ }^{\circledR}$ ).

12 I. Lagzi, B. Kowalczyk, D. Wang and B. A. Grzybowski, Angew. Chem., Int. Ed., 2010, 49, 8616-8619.

13 I. Lagzi, D. Wang, B. Kowalczyk and B. A. Grzybowski, Langmuir, 2010, 26, 13770-13772.

14 A. Z. Patashinski, R. Orlik, K. Paclawski, M. A. Ratner and B. A. Grzybowski, Soft Matter, 2012, 8, 1601-1608.

15 P. G. de Gennes, F. Brochard-Wyart and D. Quere, Capillarity and Wetting Phenomena, Springer-Verlag, New York, 2004, p. 286.

16 L. Rayleigh, Proc. London Math. Soc., 1878, s1-10, 4-13.

17 J. D. McGraw, J. Li, D. L. Tran, A. C. Shi and K. DalnokiVeress, Soft Matter, 2010, 6, 1258-1262. 